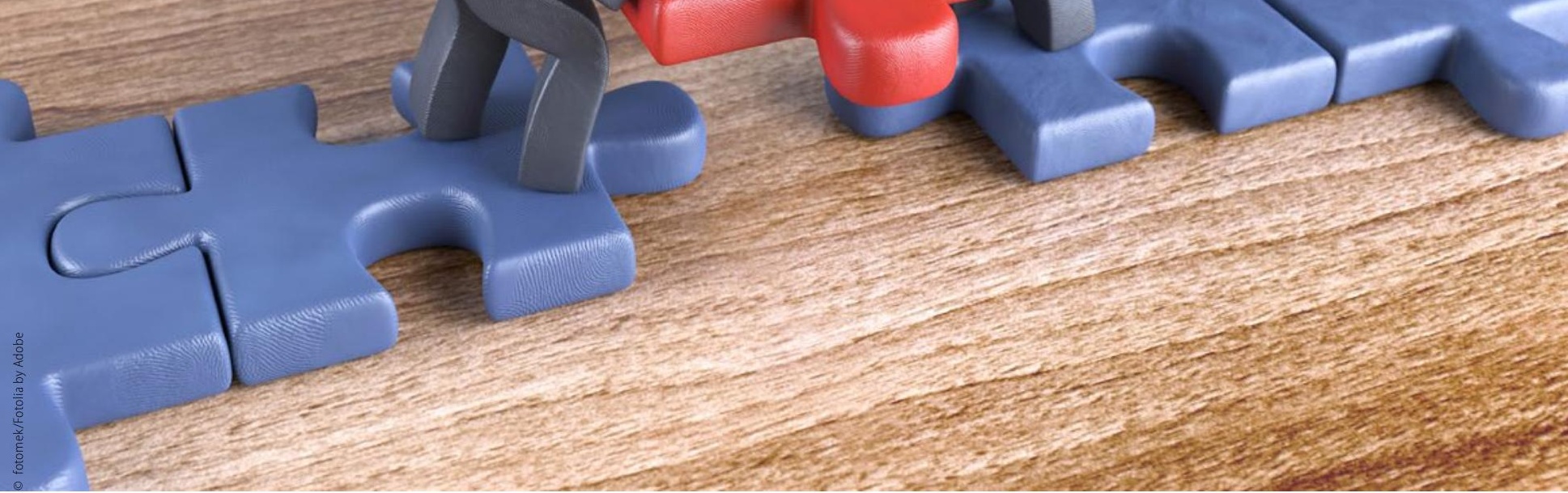

Rätselhafte Studienergebnisse

\title{
Warum es sich lohnt, die Wirkweisen eines Medikaments zu verstehen
}

Während wir einerseits von einer immer individuelleren, ganz auf den Patienten und seine genetischen und epigenitischen Besonderheiten ausgerichteten Therapie träumen, bleibt andererseits der Wunsch nach einer universellen, für viele unterschiedliche Erkrankungen wirksamen Therapie bestehen. Acetylsalicylsäure oder Statinen wurden solche Fähigkeiten zugesprochen, neuerdings stellt die Vitamin-D-Substitution eine solche Verheißung dar. Wirklich nachgewiesen wurden diese vielfältigen Effekte nie, meist blieb nur für die ursprüngliche Indikation eine nachweisbare Wirkung übrig.

\section{Wundersamer Wirkwandel der Steroide bei COPD}

In der Pneumologie waren es die inhalativen Kortikosteroide (ICS), die über ihr ursprüngliches Einsatzgebiet bei Asthma zur Standardtherapie in der COPD avisierten, obwohl nie erklärt werden konnte, wie sie eigentlich beim Lungenemphysem den Abbau von Gewebe verhindern sollen. Studien der letzten Jahre haben diesen breiten Einsatz von ICS - zu Recht - infrage gestellt, eine bessere Charakterisierung von COPD-Patienten wird hoffentlich in Zukunft dazu beitragen, ihren Stellenwert endgültig zu bestimmen. Unklar bleibt dabei, wieso praktisch alle zwischen 2000 und 2010 durchgeführten Studien deutlich po-

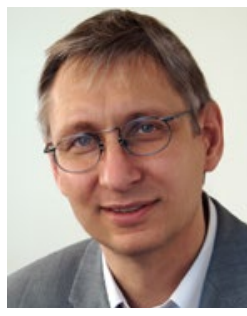

Prof. Dr. med. Tobias Welte

Klinik für Pneumologie

Medizinische Hochschule Hannover

Carl-Neuberg-Strasse 1, 30659 Hannover

welte.tobias@mh-hannover.de

sitive Effekte für ICS bei COPD zeigten, auch und gerade in wenig gut charakterisierten Patientenkollektiven, während jetzt bei nur unwesentlich geändertem Patienteneinschluss nur noch marginale Effekte nachzuweisen sind.

\section{Antibiotikum mit multiplen erwünschten Nebeneffekten?}

Nun also Azithromycin, ein Makrolidantibiotikum für das neben seinen antibiotischen Effekten verschiedene andere Wirkmechanismen beschrieben werden. Dazu gehören antiinflam- 


\section{Hier steht eine Anzeige.}

Springer 
matorische Effekte, vor allem hemmende auf TNF-alpha und Interleukin-8, aber auch eine Motilitätssteigerung des MagenDarm-Trakts und damit eine Reflux hemmende Komponente. Allerdings konnte bei keiner der im Folgenden genannten Indikationen für eine Azithromycintherapie gezeigt werden, dass die nicht antibiotischen Wirkweisen wirklich den Therapieerfolg verursachen.

\section{Einsatz bei Bronchiolitis-, Mukoviszidose, ...}

Die diffuse Panbronchiolitis, eine Erkrankung, die überwiegend in Ostasien auftritt, war die erste Erkrankung, bei der eine Dauertherapie mit Makroliden zur Anwendung kam. Zwar gibt es nur einige wenige randomisierte kontrollierte Studien zum Einsatz in dieser Indikation, die Ergebnisse waren jedoch so überzeugend, dass die Makrolidtherapie zur Standardtherapie dieser Erkrankung geworden ist.

Bei einer anderen Form der Bronchiolitis, die Bronchiolitis obliterans nach Lungentransplantation, zeigte sich ebenfalls ein Effekt des Azithromycins auf den Progress der Erkrankung. Auch hier gehört diese Therapie längst zum Standard bei chronischer Abstoßung. Die Erfahrungen aus der Bronchiolitistherapie wurden dann auf die Mukoviszidosebehandlung übertragen. Hier zeigte sich ein deutlicher Rückgang der Exazerbationsrate bei chronisch mit Pseudomonas infizierten Patienten. Das war insofern verwunderlich, als dass Makrolide ja keine antibiotische Wirksamkeit gegen diesen Erreger haben. Allerdings haben Makrolide einen Effekt auf die Fähigkeit von Pseudomonas zur Biofilmproduktion, ein Mechanismus der wesentlich dafür ist, dass der Erreger sich der körpereigenen Abwehr entzieht und der auch die Wirksamkeit von gegen Pseudomonas wirksamen Antibiotika reduziert.

Mit der „Wiederentdeckung“ der Bronchiektasenerkrankung wurden Makrolide auch hier als Dauertherapie eingesetzt, die positiven Effekte auf die Exazerbationsrate ließen sich zumindest für die mit Pseudomonas besiedelten Patienten bestätigen.

Als nächstes zeigte Azithromycin bei COPD-Patienten mit häufiger Exazerbation eine deutliche Reduktion der Exzerbationsrate, überraschenderweise vor allem bei jenen mit nur moderat eingeschränkter Lungenfunktion und nicht bei den schwerer Kranken. Nach Erregern wurde in den meisten Studien nicht systematisch gesucht, sodass unklar blieb, ob hier auch vor allem die Patienten mit einer chronischen Pseudomonasinfektion profitierten. Bronchiektasen, eine Komplikation der COPD, waren nicht ausgeschlossen, eine Häufung von Bronchiektasepatienten könnte das Ergebnis also beeinflusst haben.

\section{Dann kam Asthma bronchiale ins Spiel}

Nun also Asthma bronchiale. Im Unterschied zu den genannten Indikationen gibt es beim Asthma allerdings negative Studien, die keinen Effekt von Makroliden (oder ihrer Weiterentwicklung, den Ketoliden) auf die Exazerbationsrate zeigten. Eine kürzlich im Lancet publizierte australische Studie unterscheidet sich allerdings in mehrerer Hinsicht von den Vorstudien [1]: Sie ist größer, der Untersuchungszeitraum ist mit 48 Wochen länger als in anderen Studien und die Azithromycindosis ist höher, sie entspricht jetzt der Dosierung in den Studien mit anderer Indikation. Die Reduktion der Exazerbationsrate ist beeindruckend, eine Erklärung für diesen Effekt gibt es allerdings nicht. Bakterielle Erreger spielen ja beim Asthma kaum eine Rolle, antiinflammatorische Effekte ließen sich nicht nachweisen, Azi-

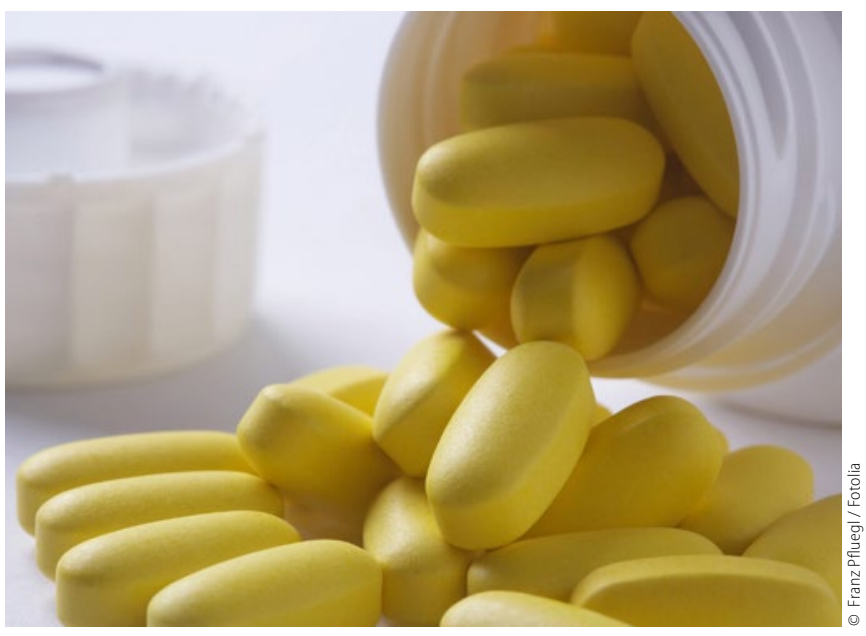

Um die Effekte bei langfristiger Einnahme von Azithromycin bei Asthma und anderen Indikationen sicher einordnen zu können, weiß man noch zu wenig über seine Wirkmechanismen.

thromycin wirkte sowohl bei eosinophilem als auch bei neutrophilem Asthma. Anders als beispielsweise bei der COPD konnten keine Nebenwirkungen wie Taubheit oder Herzrhythmusstörungen und keine Effekte auf die Resistenzentwicklung von Keimen der Rachenflora nachgewiesen werden.

Gerade weil der Wirkungsmechanismus dieser Therapie so unklar ist, fällt es aber schwer, ihr einen Platz in der Asthmatherapie zuzuweisen. Im Editorial der Lancet-Ausgabe vom 12. August ordnen Brusselle und Pavord sie als eine Art „LetzteChance-Therapie" bei sonst nicht kontrollierbarem Asthma ein. Sie betonen aber auch, dass sie gegenüber vielen heute eingesetzten Therapien wie den neuen Biologicals erhebliche Kostenvorteile hat.

\section{Aus Nutzen kann schnell Schaden werden}

Die hier geschilderte Geschichte des Azithromycins zeigt, wie schwierig die Einordnung, aber auch die Weiterentwicklung von Medikamenten ist, deren Wirkweise man nicht wirklich versteht. Ich bin persönlich nicht davon überzeugt, dass Makrolidantibiotika so nebenwirkungsarm sind, wie es in der australischen Studie scheint. Die Patientenzahlen aus allen aufgezählten Studien sind viel zu klein, als dass man wirklich Effekte wie eine erhöhte kardiovaskuläre Sterblichkeit erkennen könnte. Bei unkritischem Einsatz kann aus einem Nutzen dann schnell ein Schaden werden. Inhalative Makrolide würden die Nebenwirkungsraten wahrscheinlich reduzieren. Es ist schwer zu verstehen, warum eine solche Entwicklung, die technisch anspruchsvoll, aber nicht unmöglich erscheint, nicht begonnen wird.

Auf jeden Fall macht die wissenschaftliche Beschäftigung mit diesen Substanzen Sinn. Denn am Ende würde ich mich wohler fühlen, wenn ich verstehen würde, warum die Substanzen wirken. Sonst verschwinden die Effekte über die Zeit - wie bei so vielen anderen Wundermitteln.

\section{Literatur:}

1. Gibson PG, Yang IA, Upham JW et al. Effect of azithromycin on asthma exacerbations and quality of life in adults with persistent uncontrolled asthma (AMAZES): a randomised, double-blind, placebo-controlled trial. Lancet. 2017 Aug 12;390(10095):659-668. doi: 10.1016/S0140-6736(17)31281-3. 